\title{
12 Nutzerorientierung als Leitgedanke von technologischen Innovationen im Gesundheitswesen
}

\author{
Christoph Dockweiler
}

\section{Bestandsaufnahme und Stellenwert}

Der Einsatz von Informations- und Kommunikationstechnologien in der medizinischen und pflegerischen Versorgung erfährt national als auch international eine bemerkenswerte Aufmerksamkeit. In Europa hat hierzu auch die strategische Förderung der Europäischen Kommission im Rahmen des „eHealth Action Plan 2012-2020“ beigetragen (European Commission 2014). Das Rahmenprogramm verfolgt das Ziel einer grundlegenden Verbesserung der Gesundheit der Bürger durch die digitale Bereitstellung lebenswichtiger Informationen über Ländergrenzen hinweg. Ebenso sollen zukünftig durch die Einbeziehung von digitalen Technologien in die medizinische und pflegerische Versorgung die Qualität und vor allem die Zugänglichkeit verbessert werden. Als Kernelement der bereits begonnenen digitalen Transformation im Gesundheitswesen betrachtet die Europäische Kommission die Schaffung (be-) nutzerfreundlicher und umfassend akzeptierter elektronischer Gesundheitsdienste durch die Einbeziehung von Professionals und Patienten innerhalb von Strategien, Entwicklungsprozessen, der Gestaltung von Technik und Forschung sowie der Umsetzung in der Praxis.

\section{Beschreibung des Transformationsprozesses}

Neben der klaren Positionierung der Kommission zu einer stärkeren Berücksichtigung der Nutzerperspektiven lässt ebenso die bisher noch stockende Implementierung von E-Health-Anwendungen in der Versorgung - im Vergleich zum zweiten 
Gesundheitsmarkt - den Schluss zu, dass Forschung und Entwicklung nicht (maßgeblich) „top down“ erfolgen können. Denn Fragen einer bedarfs- und bedürfnisgerechten (oder gar diversitätssensiblen) Einführung und Nutzung von Versorgungstechnologien lassen sich nicht allein durch die Prämisse des „technisch Möglichen“ lösen, sondern müssen sich viel mehr am „technisch Notwendigen“ orientieren. Die Diskussion „technischer“ Aspekte (z.B. technologische Interoperabilität) ist zwar ein notwendiger Bestandteil der gesellschaftlichen Diffusion von Technologien, und natürlich muss auch eine bedarfsadäquate (und standardisierte) Technikinfrastruktur geschaffen werden. Doch erscheint diese Engführung bisher mehr als Diskussion über als mit den Nutzern. Eine Diskussion, die eben auch dem Gedanken der „sozialen und kulturellen Interoperabilität" folgen muss. Immer zahlreicher werdende Arbeiten setzen sich infolgedessen mit der Frage der Diffusion gesundheitstelematischer Systeme innerhalb verschiedener gesellschaftlicher Teilbereiche auseinander (Dockweiler 2016). Der Rückgriff auf die spezifischen individuellen (z.B. Technikkompetenzen, Anforderungen an und Einstellungen gegenüber Technik) und strukturellen Gegebenheiten (z.B. Technikinfrastruktur, Lebensumfeld der Patienten) innerhalb der jeweiligen Settings, in denen technische Innovationen wirksam werden sollen, stellt dabei ein zentrales Merkmal gelingender Implementationsprozesse dar.

\section{Chancen und Risiken}

Aspekte der Nutzerorientierung und die Förderung von technologischen Innovationsprozessen bedingen sich dabei wechselseitig. Sie sind gleichzeitig Chance und Risiko. Innovative Gesundheitstechnologien zeigen einerseits ein beachtliches Potenzial für eine größere Nutzerorientierung im Sinne der Individualisierung von Gesundheitsleistungen. Andererseits tangieren der Prozess der Digitalisierung von Gesundheit und die Ausschöpfung der inhärenten Potenziale in zentraler Weise die praktische Diffusion innovativer Versorgungsmaßnahmen und -strukturen sowie die dabei zentralen Fragen der Nutzerorientierung, der Akzeptanz sowie der Partizipation. Diese sind, unter der Annahme einer evidenten Steigerung der Qualität und Kosten-Effektivität durch den Einsatz elektronischer Gesundheitsdienste, durchaus grundsätzlicher Natur. Denn werden Prozesse der Entwicklung und Implementierung nicht unter diesen Gesichtspunkten begleitet und findet darauf aufbauend keine Ableitung von akzeptanzfördernden Maßnahmen statt, bleibt das Potenzial innovativer Technologien im Gesundheitswesen ungenutzt. Dabei bieten verschiedene thematische Bezugsdisziplinen zielführende Anknüpfungspunkte zur Förderung einer deutlicheren Nutzerorientierung.

In den Gesundheitswissenschaften und der Medizin ist das Paradigma der Patientenorientierung (in Anlehnung an den Terminus der Nutzerorientierung) als Leitbild der (Fort-)Entwicklung von Gesundheitsförderung, Prävention und Versorgung prominent verankert. Geprägt wurde der Nutzerbegriff im deutschen Gesundheitswesen durch den Sachverständigenrat zur Begutachtung der Entwicklung im Gesundheitswesen, der 2001 erstmals von Nutzern sprach, die in unterschiedlichen Rollen (z.B. als versierter, mündiger Kunde) dem Gesundheitssystem gegenüberstehen und es entlang ihrer Bedürfnisse kompetent „nutzen“. Ein Verständnis, welches keineswegs frei von inkongruenten Erwartungshaltungen war und heute noch ist: Die Person tritt als interessierter, informierter Bürger auf, als kompetenter Koproduzent seiner 
eigenen Gesundheit, als versierter Kunde, als Mitglied sozialer Gemeinschaften, die innerhalb von Selbsthilfe kooperieren, demgegenüber als hilfsbedürftiger, leidender Patient (Ewert 2012). Dabei zeigt sich Nutzerorientierung als Leitgedanke, welcher die subjektiven Bedarfe und Bedürfnisse, Erfahrungen, Haltungen und Bewertungen der Nutzer von Versorgung in den Mittelpunkt des handlungsleitenden Forschungsinteresses stellt, um sie als zentrale Ziel- und Korrekturdimension in das lernende System Gesundheitswesen einzuspeisen.

Eine hierzu sehr ähnliche, zentrale und bis heute persistente Erkenntnis sozialwissenschaftlicher Technikforschung ist das Verständnis von technologischer Entwicklung als sozialer Prozess (Decker et al. 2012). Die beiden relevanten Forschungslinien der Technikbedarfs- und der Technikinnovationsforschung fragen einerseits nach dem Informationsbedarf von Nutzern (also der Ausprägung von Wissensständen und technikbezogenen Kompetenzen und daraus resultierenden Bedürfnissen) und den individuellen Vorstellungen der zukünftigen Technikintegration. Insbesondere die Technikinnovationsforschung befasst sich dabei mit den Leitbildern für Technikentwicklung, die nutzerorientierten Technikanforderungen gerecht werden, und mit den notwendigen Kooperationsbeziehungen zwischen spezialisierten Technikanbietern und Nutzern, um einen gemeinsamen Wertschöpfungsprozess zu organisieren.

Ebenso finden sich relevante Beispiele zur Nutzerorientierung in der Informatik. Die Requirements-Forschung integriert dabei jene Aktivitäten, bei denen es um das Erfassen, Dokumentieren und Verwalten von Anforderungen der verschiedenen Interessengruppen geht (Kroll et al. 2003).

\section{Trends und Entwicklung}

Wenn also die Rede von Nutzerorientierung als Leitgedanke (sozio-)technischer Innovationen ist (und nichts anderes sind sie, da technische Innovationen nicht losgelöst von der sozialen Aneignung und Nutzung innerhalb gesundheitsbezogener Kontexte betrachtet werden können), bedeutet dies die Analyseebene nutzerseitiger Einflussfaktoren auf die Entwicklung, Gestaltung und Implementierung derartiger Technologien in besonderer Weise zu berücksichtigen. Dazu gehört nicht nur die Explikation der Bedarfe, Bedürfnisse und Wahrnehmungen von Innovationen, sondern auch die Analyse des Umgangs der Nutzer mit den Technologien in den Praxissettings. Hierzu gehört etwa die Erfassung von individuellem Wissen und Kompetenzen, die für eine adäquate Nutzung erforderlich sind. Auf dieser Basis kann die Etablierung von Empowermentprozessen auf der Mikroebene (z.B. in Form von Schulung, Information, Beteiligung), der Mesoebene (z.B. Schulungen, institutionelle Beteiligungsformen) und der Makroebene (z.B. Kampagnen mit dem Ziel der Aufklärung, Wissenssteigerung und Problemsensibilisierung) vorangetrieben werden.

Nutzerorientierung bedeutet damit auch den Blick von den unmittelbaren Nutzern auf die Rahmenbedingungen der Nutzung zu lenken und die vorliegenden Strukturen und Prozesse hinsichtlich ihrer nutzenerleichternden Eigenschaften zu analysieren. Dies können Unterstützungs- und Bildungsstrukturen sein, die Fortentwicklung datenschutzrechtlicher Rahmungen aus der Perspektive des mündigen Bürgers im Sinne der informationellen Selbstbestimmung, berufsrechtliche Bedingungen, die das rechtssichere Handeln der Professionals unterstützen, aber auch die fortwähren- 
de ethische Reflexion der Digitalisierung unserer Gesundheit und unserer Lebenswelten, in denen Gesundheit (re-)produziert wird.

Dabei verlangen derartige Interventionsfelder einen konsequenten inter- und transdisziplinären Diskurs zwischen Wissenschaft, Politik, Praxis und Wirtschaft, der möglichst frühzeitig in der Planung und Entwicklung von Innovationen ansetzt. Dazu gehören innovative Formen der Kooperation zwischen Praxis und Wissenschaft, die Koproduktion von Wissen sowie die Partizipation relevanter Nutzergruppen in Forschung und Entwicklung (Bergold u. Thomas 2012). Durch die Ermöglichung von Partizipation kann der notwendige gegenseitige Lernprozess der verschiedenen Akteure gefördert werden. Ebenso können die vorliegende Meinungs- und Interessenvielfalt besser im Rahmen von Forschung und Entwicklung berücksichtigt und potenzielle Konflikte bei der Implementation innovativer Versorgungskonzepte bereits im Vorfeld reduziert werden. Die Partizipation im Rahmen von Forschung und Entwicklung stellt damit einen relevanten Schritt zu mehr Bedarfs- und Bedürfnisgerechtigkeit in der Versorgung unterschiedlicher Bevölkerungs- und Patientengruppen dar.

Allerdings betrachten erst wenige Beiträge das komplexe Geflecht unterschiedlicher Bedingungen der Nutzerorientierung im Bereich innovativer Gesundheitstechnologien im Sinne sozialwissenschaftlicher Ansätze der Akzeptanzforschung - und das obwohl die Bereitschaft zur Nutzung einer technologischen Versorgungsinnovation explizit mit der Frage verknüpft ist, wie diese aus der Perspektive der Nutzer vor dem Hintergrund der vorliegenden Bedarfe, Bedürfnisse und Rahmenbedingungen wahrgenommen wird.

\section{Literatur}

Bergold I, Thomas S (2012) Partizipative Forschungsmethoden: Ein methodischer Ansatz in Bewegung, in: Forum Qualitative Social Research, Ig. 13, Nr. 1, Art. 30. Stand: 22.04.2017.URL: http://www.qualitativeresearch.net/index.php/fqs/article/view/1801/3332 (abgerufen am 23.06.2017)

Decker M, Grundwald A, Knapp M (2012) Der Systemblick auf Innovationen. Technikfolgenabschätzung in der Technikgestaltung. edition sigma Verlag Berlin

Dockweiler C (2016) Akzeptanz der Telemedizin. In: Fischer F, Krämer A (Hrsg.) eHealth in Deutschland, 257-271, Springer Verlag Berlin

European Commission (2014) Putting patients in the driving seat: A digital future for healthcare. European Commission (Hrsg.) Brussels

Ewert B (2012) Nutzer im Gesundheitswesen: Koproduzenten zwischen Autonomieansprüchen, Kompetenzanforderungen und Verunsicherung. WSI Mitteilungen. Ig. 3. Stand: 22.04.2017. URL: http://www.boeckler. de/wsimit_2012_03_ewert.pdf (abgerufen am 23.06.2017)

Kroll P, Kruchten P, Booch G (2003) The Rational Unified Process Made Easy: A Practitioner's Guide to the RUP, Addison-Wesley Longman Publishing Amsterdam 